\title{
EXPERIMENTELLE ERGEBNISSE ÜBER DIE BEDEUTUNG DER HETEROSIS BEI DROSOPHILA*
}

\author{
D. SPERLICH \\ Institut fur allgemeine Biologie, \\ Schwarzspanierstrasse I7, A-rogo, Wien IX, Osterreich
}

\section{ZUSAMMENFASSUNG}

In natürlichen Populationen von Drosophila, einer panmiktischen und sich sexuell reproduzierenden Art, spielt Heterosis eine grosse Rolle. Es besteht, wie zumindest für den Inversionspolymorphismus gezeigt werden konnte, ein balanciertes System und die Hãufigkeit der koexistierenden chromosomalen Strukturen ist im Gleichgewicht. Dieser Gleichgewichtszustand wird durch die Heterosis aufrechterhalten und die chromosomalen Strukturtypen sind koadaptiert. In bezug auf die synthetischen Letalfaktoren und die Nicht-Zufalls-Assoziation von Inversionen bestehen deutliche, nicht-allelische Genwechselwirkungen. Biochemische Untersuchungen zeigen, dass in natürlichen Populationen von Drosophila $40 \%$ aller Genloci polymorph sind und es erscheint daher in der Regel ein balanciertes Populationssystem vorzuliegen. Experimentalpopulationen mit künstlich reduzierter genetischer Variabilitat befinden sich in einem ungewōhnlichen Zustand und streben danach ihre frühere Heterozygotie wieder zu erlangen. Fremde Chromosomen werden daher leicht in den Genpool solcher Populationen eingebaut. Heterozygote Populationen hingegen sind gepuffert und fremdes genetisches Material wird rasch eliminiert. Ebenso dürften sich auch strahleninduzierte und spontane Letalmutationen verhalten. Für den Züchter könnten diese Ergebnisse von einigem Interesse sein. Es erscheint danach leicht neues genetisches Material in Inzuchtlinien einzuführen, in Kreuzungszuchten hingegen sollte es rasch wieder eliminiert werden.

Die Bedeutung der Heterosis für die Zusammensetzung des Genpools natürlicher Populationem von Drosophila wurde vor allem von DoBzhansky und seiner Schule untersucht, und die Balance-Hypothese zur Erklärung der genetischen Struktur der Populationen aufgestellt (s.z.B. WALIACE, I968). Diese Hypothese nimmt an, dass die genetische Mannigfaltigkeit natürlicher Populationen dadurch aufrecht erhalten wird, dass die Heterozygoten durch die natürliche Selektion am meisten begünstigt sind. Dadurch kommt es zu einer Balance in der relativen Häufigkeit der Allele eines Gens, die nur von der Fitness der benachteiligten Homozygoten bestimmt wird. Dass eine solche Annahme zumindest in manchen Fällen stimmt, konnte zunächst für die Aufrechterhaltung des Inversionspolymorphismus bei Drosophila bewiesen werden. In natürlichen

(*) Offizielle Berichterstattung über die Studientagung der Europäischen Vereinigung für Tierzucht, Kommission für Haustiergenetik, Gödöllö, Ungarn, 24. August r97o. 
Populationem von vielen Drosophilaarten finden sich zahlreiche und oft auch komplexe Strukturveränderungen der Chromosomen, die sich an den Riesenchromosomen von Drosophila leicht direkt als Inversionen erkennen lassen. In strukturpolymorphen Populationen koexistieren also von jedem Chromosom mehrere verschiedene chromosomale Strukturtypen. Die relative Häufigkeit derselben ist in verschiedenen lokalen Populationen unterschiedlich. Für Drosophila ist es leicht möglich, relativ grosse Populationen experimentell zu halten und so die dynamischen Prozesse im Genpool ständig zu kontrollieren. Experimentalpopulationen, die mit verschiedenen, von natürlichen Verhältnissen abweichenden Frequenzen der chromosomalen Strukturtypen künstlich begonnen werden, verändern ihre anfängliche Zusammensetzung solange, bis eine bestimmte Gleichgewichtssituation erreicht wird. Werden die Experimentalbedingungen konstant gehalten und die chromosomalen Strukturtypen aus derselben natürlichen Population genommen, so stellt sich unabhängig von der Anfangszusammensetzung stets dieselbe Häufigkeitsverteilung ein, wenn der Gleichgewichtszustand erreicht wird (Wright u. Dobzhansky, I946; Dobzhansky, I95I). Ein solches Verhalten ist nur durch Heterosis zu erklären. Tatsächlich konnte die Überlegenheit der Strukturheterozygoten in bezug auf viele Fitnessmerkmale wie Ei-Imago-Überlebensrate, Fruchtbarkeit, sexuelle Aktivität, Lebensdauer, Entwicklungsgeschwindigkeit u. a. direkt nachgewiesen werden. (s. Sperilich, I967b, Spiess, I968). Gleichzeitug wurde es immer mehr klar, dass nicht die Inversionen selbst, sondern die in ihnen eingeschlossenen Gene den Heterosiseffekt bedingen (Dobzhansky, I948). Die Heterosis der Strukturheterozygoten ist eine echte Überdominanz (Euheterosis) und geht von einer ganzen Gengruppe aus (Dobzhansky, r952). Die Genblöcke des einen Strukturtyps sind. an die des oder der anderen im Sinne eines maximalen Heterosiseffektes koadaptiert. Die natürliche Selektion hat im Laufe der Evolution dieser Art offensichtlich im Sinne einer Erhöhung der Heterosis gewirkt, also die Kombinationsfähigkeit der Chromosomen verbessert. Die Inversion selbst hat dabei nur als Crossingover-Verhinderer gewirkt, der die Genblöcke zusammengehalten hat.

Nachdem der Inversionspolymorphismus der Drosophila-Arten als balancierter Polymorphismus erkannt war, ergab sich als weitere Frage, ob HeterosisSysteme auch für die Genzusammensetzung von Arten von Bedeutung sind, die keine Inversionen besitzen. Zunächst stellte es sich heraus, dass natürliche Populationen eine grosse genetische Bürde in Form von rezessiven Letalfaktoren, Semiletal-, Subvitalitäts- und Sterilitätsfaktoren besitzen. Im Durchschnitt tragen etwa $30 \%$ aller Chromosomen einen rezessiven Letalfaktor. Die Frage, ob diese genetische Bürde durch Mutation zu erklären ist, oder aber die Häufigkeit der Detrimentalfaktoren vorwiegend durch ihre Heterosis bedingt wird, ist ein bis heute viel diskutiertes, aber nicht gelöstes Problem (WALLACE und DoBzHANSKy, I 962). Soviel steht heute fest, dass sicherlich beide Möglichkeiten bestehen und die Frage muss nun genauer so gestellt werden : Ist die rezessive verborgene Bürde vorwiegend eine Mutations-oder eine balancierte Bürde? Crow et al. (MORTON Crow u. Mulier, I956; Crow u. Temin, I964) sind der Meinung, dass in natürlichen Populationen vorwiegend eine Mutationsbelastung vorliegt, während 
Dobzhansky u.a. der Meinung sind, dass vorwiegend eine balancierte Bürde vorliegt. Die Entscheidung ist für das Verständnis des Aufbaus natürlicher Populationen von Bedeutung.

Grosse Schwierigkeiten bereitet in diesem Zusammenhang die von vielen übersehene Tatsache, dass Gene niemals alleine wirken, sondern nur mit anderen zusammen. Ein-Gen-Heterosis konnte wohl für das Sichelzellen-Gen beim Menschen bewiesen werden (ALLISON, 1956), aber sonst scheint sie relativ selten zu sein. Bei Drosophila lassen sich alle Fälle von Ein-Gen-Heterosis auch durch Koppelung mit anderen Fitnessgenen erklären. Es kann angenommen werden, dass die Heterosis oft von Genblöcken ausgeht und nicht-allelische Genwechselwirkungen von Bedeutung sind. Wie Dobzhansky und Spassky (I960) zeigen konnten, kann durch Kombination von sonst nicht auffällig Fitness-reduzierenden Genen eine Letalwirkung eintreten, die als synthetische Letalwirkung bezeichnet wird. Umgekehrt ist es uns gelungen, durch Kombination zweier Letalfaktoren in einem Chromosom eine Aufhebung des Letal-effektes zu erhalten. Gibson u. Thoday (I962) fanden, dass die Kombination zweier Loci, die die Sternopleuralborstenzahl bei $D$. melanogaster erhöhen, letal wirkt. Semiletalfaktoren des X-Chromosoms bewirken, dass in bestimmten Stämmen von D. melanogaster die Zahl der Männchen sehr gering ist. Bei längerer Laborhaltung wird, wie KARLIK (I970) zeigen konnte, die Semiletalwirkung des X-Chromosoms immer geringer und die Männchenfrequenz steigt auf $50 \%$ an. Die Verbesserung der X-Chromosomen geht wohl kaum auf Rückmutation am Semiletallocus, sondern teilweise auf Wechselwirkung zwischen Neumutationen und dem Semiletalfaktor zurück, oder auf die selektive Anhäufung von Faktoren im genetischen Hintergrund, die die nachteilige Wirkung des Semiletalfaktors aufheben. Auch in unseren Untersuchungen über den Inversionspolymorphismus von $D$. subobscura (SPERLICH, I967a) war es uns aufgefallendass unabhängige Inversionen stets nur in Cis- oder Transtellung vorkommen. Eine genaue Analyse ergab, dass fast in allen Fällen ein vollständiges AustauschUngleichgewicht $z$ wischen den Inversionen natiurlicher Populationen bestand. Das bedeutet, dass bestimmte Austauschkombinationen (zwischen A, $a$ und B, $b$ sind etwa $\mathrm{AB}, a \mathrm{~B}, \mathrm{~A} b$ und $\mathrm{ab}$ möglich) nicht auftreten (etwa $a \mathrm{~B}$ und $\mathrm{A} b$ ) (SPERLICH und FEUERBACH, I969). Es muss hier an eine starke Wechselwirkung gedacht werden, die in einem Fall selektiv begünstigt, im anderen selektiv benachteiligt. Für einen Fall konnten FEUERBACH-MRAVLAG und SPERLICH (I970) nachweisen, dass die Kombination zweier Inversionen in einem Chromosom in Cis-Stellung dominant letal ist. Wir müssen also die Frage um die genetische Bürde auch im Zusammenhang mit der zweifellos bestehenden, nicht-allelischen Genwechselwirkung sehen. Es ist verständlich, dass eine Neumutation in dem einen genetischen Milieu nachteilig, im anderen vorteilhaft sein kann. Worauf die Genwechselwirkung bei höheren Organismen zurückgeführt werden kann, ist noch weitgehend unklar und wird auch in jedem Fall speziell zu untersuchen sein. Aus der Molekulargenetik kennen wir die Regulationsmechanismen : Regulatorgen Operatorgen - Operon. Bei Drosophila konnten BeERMANN (I952), BECKER (I962), KRÖGER (I963) u. a. zeigen, dass bestimmte Gengruppen der Riesenchromosomen zu bestimmten Zeiten der Larvenentwicklung aufgepufft sind und mRNS produzieren. In anderen Phasen der Entwicklung hingegen sind diese Orte nicht 
aktiv. Es besteht also auch hier eine Regulation, die für bestimmte Fälle mit dem Verpuppungshormon Ecdyson in Verbindung steht. Ob dieses Hormon selbst als Repressor angesehen werden kann, ist jedoch fraglich. Sicherlich sind Genwechselwirkungen solcher Art auch bei höheren Organismen denkbar, doch können auch andere Modelle, die sich hinter Epistasis und Suppression verstecken, gedacht werden.

Lewontin und Hubby (1966) haben an D. pseudoobscura Untersuchungen angestellt, die von der Annahme ausgingen, dass Eiweissvariationen gleichzusetzen sind mit genetischer Variation eines Strukturgens. Sie griffen I8 verschiedene Eiweiss- oder Enzym-Eiweiss-Systeme, die elektrophoretisch dargestellt werden können, heraus und fanden, dass davon $7 \mathrm{mehr}$ als eine Variante in natürlichen Populationen zeigten, also genisch polymorph waren. Das bedeutet, dass etwa $40 \%$ aller Genloci in natürlichen Populationen polymorph sind, mit einer Allelhäufigkeit die Mutation allein als Quelle der Variabilität ausschliesst. Darüber hinaus erwies es sich, dass bestimmte Eiweissvariationen mit bestimmten Inversionsstrukturen dieser Drosophilaart positiv korreliert waren. Daraus ergibt sich, dass auch die mit molekularen Methoden durchgeführten Analysen die Annahme der koadaptiven und komplexen Heterosiswirkung unterstïtzen. Jedenfalls kann ein derartiges Populationssystem nur mit einer Art Balance erklärt werden.

Wenn die normale Populationsstruktur panmiktischer Organismen (oder solcher, deren Inzuchttendenz gering ist) eine polymorphe ist, in der einfache oder komplexe Heterosissysteme eine Rolle spielen, muss die genetisch homozygote Struktur einen Ausnahmezustand darstellen. WALLACE (I958, I963) fand bei D. melanogaster, dass Neumutationen in vollkommen homozygoten Individuen im Durchschnitt heterotisch, also vorteilhaft sind. In bereits ausgewogen heterozygoten Individuen hingegen sind Neumutationen im Durchschnitt nachteilig. Die Untersuchungen mit Drosophila kommen an den Zustand vollkommener Homozygotie, zumindestens für ein Chromosom, relativ nahe heran, da sogenannte Markierungschromosomen mit Crossing-over-Verhinderern es erlauben, im Kreuzungsgang identische Replikas eines ursprünglichen Chromosoms wieder zu kombinieren. Inzucht allein kommt dem vollkommen homozygoten Zustand nur theoretisch sehr nahe, während in der Praxis oft die natürliche Selektion der Inzucht die Blance halten kann.

In unserem Laboratorium in Wien haben wir in diesem Zusammenhang Untersuchungen durchgeführt, über die hier noch kurz berichtet werden soll. Der Grundgedanke war der, dass bei Arten, deren natürliche Populationsstruktur eine polymorphe, also genetisch weitgehend heterogene ist, die künstliche Veränderung der Population im Sinne einer zunehmenden Homozygotie eine abnorme Situation schafft, in der eine Rückkehr zur früheren Heterozygotie selektiv begünstigt ist. Dafür stehen der Population zwei Wege offen :

I. Die Aufnahme von neuer, genetischer Variabilität aus zufliessendem Genmaterial in den Genpool offener Populationen (CARson, I96r).

2. Die Aufnahme von Neumutationen in den Genpool. Es wäre zu erwarten, dass Populationen im Heterosisgleichgewicht fremdes, genetisches Material und Neumutationen nur selten in den Genpool einbauen, sondern sie rasch wieder 
entfernen. Homozygot gemachte Populationen hingegen wären ungesättigt und sollten Neumutationen oder fremde Allele wie " ein Schwamm " (TSCHETWERIKoFf, I926) aufsaugen.

Unsere Untersuchungen mit D. melanogaster wurden so durchgeführt, dass einzelne Linien mit Hilfe von Markierungsstämmen für das II-Chromosom vollständig homozygot gemacht wurden, andere Linien aber die natürliche, polymorphe Genpoolzusammensetzung hatten. Von diesen Linien wurden « homozygote (monochromosomale) " und "heterozygote (polychromosomale) " Experimentalpopulationen abgeleitet und fremdes genetisches Material in Form von Chromosomen, die mit dominanten Genen markiert waren, eingeführt. Mit grosser Regelhaftigkeit zeigte es sich, dass diese fremden Chromosomen von monochromosomalen Populationen akzeptiert wurden und daher eine Gleichgewichtshäufigkeit erreichten, während polychromosomale Populationen die fremden Chromosomen stets rasch eliminierten (SPERLICH u. KARLIK, I968, I970). Untersuchungen über das Schicksal von röntgeninduzierten und spontanen Letalmutationen in homound heterozygoten Populationen werden derzeit von Mrs. MuKhERJEe und von KARLIK durchgeführt. Soweit vorläufige Experimente uns zeigen, werden Letalfaktoren aus polychromosomalen Populationen viel rascher entfernt, als aus monochromosomalen. Sollten sich diese Ergebnisse verallgemeinern lassen, so scheinen ihre Konsequenzen auch züchterisch interessant. Genetisch heterogene Zuchtlinien erscheinen in bezug auf die Wirkung der natürlichen Selektion besser gepuffert (LERNER, I954). Sie nehmen Neumutationen kaum in ihren Genpool auf und gelegentliche Einkreuzung mit Individuen fremder Populationen werden nur beschränkt zu einer dauerhaften Inkorporation von fremden Genen führen. Andererseits scheinen Inzuchtlinien besonders empfindlich, wenn es darum geht, eine Anpassung des Genpools an ganz neue Verhältnisse zu erreichen. Es wird hier relativ leichter sein, neues genetisches Material, sei es als Neumutation, sei es als eingekreuztes Genmaterial in den Genpool der Population einzufiihren.

Reçu pour publication en novembre 1970.

\title{
RÉSUMÉ
}

\author{
RÉSULTATS D'EXPÉRIENCES \\ SUR L'IMPORTANCE DE L'HÉTÉROSIS CHEZ IA DROSOPHIIE
}

Dans les populations naturelles de Drosophiles, espèce à reproduction sexuée et exogame, l'hétérosis joue un rôle important. Il a pu être démontré qu'il existe, pour le polymorphisme d'inversion, un système judicieusement équilibré, avec équilibre de la fréquence des arrangements des gènes coexistants. Cet état d'équilibre est dû à l'hétérosis, et les arrangements de gènes sont bien adaptés entre eux. Pour des combinaisons planifiées d'inversions léthales indépendantes, les interactions entre gènes non-alléliques sont fréquentes. Les recherches biochimiques montrent que, dans les populations naturelles de Drosophiles, 40 p. 100 de tous les locis sont polymorphes et que la structure de la population constitue en règle générale un système équilibré. Les populations expérimentales, avec une variabilité génétique réduite artificiellement, se trouvent dans des conditions insolites et ont tendance à regagner leur degré précédent d'hétérozygotie. Elles assimilent très facilement les chromosomes étrangers. Par contre, les populations hétérozygotes 
sont "tamponnées " et éliminent très vite les gènes étrangers. Ce même phénomène semble se produire aussi bien pour les mutations léthales spontanées que pour celles provoquées par les rayons $X$. Cela signifie, pour les éleveurs, que, si un nouveau matériel génétique peut aisément être introduit dans des lignées consanguines, dans les lignées non consanguines, par contre, un tel matériel risque d'être éliminé rapidement.

\section{SUMMARY}

\section{EXPERIMENTAL RESULTS ON THE IMPORTANCE OF HETEROSIS IN DROSOPHILA}

In natural populations of Drosophila, an outbreeding and sexual reproducing species, heterosis plays an important role. As could be shown for inversionpolymorphism a balanced system exists in which the frequency of the coexisting gene arrangements is at equilibrium. This equilibrium is due to heterosis and the gene arrangements are coadapted. For synthetic lethals and nonrandom association of independent inversions, non-allelic gene interactions are frequent. Biochemical investigations reveal that 40 per cent of all loci are polymorphic in natural populations of Drosophila and the balanced system of population structure seems to be the rule. Experimental populations with artificially reduced genetic variability are in an extraordinary condition and tend to regain their former degree of heterozygosity. They incorporate alien chromosomes very easily. Heterozygous populations, however, are buffered and eliminate alien gene material very rapidly. The same seems to be true for $\mathrm{X}$-ray induced and spontaneous lethal mutations. For breeders these findings could be of some interest. It may be that new genetic material can easily be introduced in inbred lines, in outbred strains, however, it is expected to be eliminated very rapidly.

\section{LITERATUR}

Allison A.C., I956. Population genetics of abnormal human haemoglobins. Genetica, 6, 430-434. BECKER H.J., 1962. Die Puffs der Speicheldrüsenchromosomen von Drosophila melanogaster II. Chromosoma, 13, 341-384.

BEERMAN W., I952. Chromosomenkonstanz und spezifische Modifikationen der Chromosomenstruktur in der Entwicklung und Organdifferenzierung von Chironomus tentans. Chromosoma, 5, I39-198.

Crow J.F., TEMIN R.G., I964. Evidence for the partial dominance of recessive lethal genes in natural populations of Drosophila. Amer. Nat., 98, 2I-33.

CARSON H., I96r. Relative fitness of genetically open and closed experimental populations of Drosophila robusta. Genetics, 46, 553-567.

DoBzHANSKy Th., I 948 . Genetics of natural populations. XVIII. Experiments on chromosomes of Drosophila pseudoobscura from different geographic regions. Genetics, 33, 588-602.

Dobzhansky Th., I95ז. Genetics and the Origin of Species. Columbia Univ. Press, New York.

DOBZHANSKY Th., I952. Nature and origin of heterosis. In: Heterosis (Ed. J. Gowen). Jowa State College Press, Ames.

Dobzhansky Th., Spassky B., I960. Release of genetic variability through recombination. V. Break up of synthetic lethals by crossing over in Drosophila pseudoobscura. Zool. Jahrb., 88, 57-66.

Feuerbach-Mravlag H., Sperich D., Ig7r. In Vorbereitung zum Druck.

Gibson J.B., THODAY J.M., I962. Effects of disruptive selection. VI. A second chromosome polymorphism. Hevedity, 17, I-26.

KARLIK A. Untersuchungen über polygene Supressorwirkung auf Semiletalfaktoren des X-Chromosoms von Drosophila melanogaster. In Vorbereitung zum Druck.

KRöGER H., r963. Experiments on the extranuclear control of gene activity in dipteran polytene chromosomes. J. cell. Comp. Physiol., 62, (Supp1. I) 45-59. 
LERNER I.M., I954. Genetic Homeostasis, Oliver and Boyd, Edinburgh.

L.EWONTIN R.C., HubBY J.I., r966. A molecular approach to the study of genetic heterozygosity in natural populations. II. Amount of variation and degree of heterozygosity in natural populations of Drosophila pseudoobscura. Genetics, 54, 595-609.

Morton N., Crow J.F., Muller H.J., I956. An estimate of the mutational demage in man from data on consanguineous marriages. Proc. Acad. Sci., (Wash.) 42, 855-863.

SPERLICH D., I 9679 . Die Bedeutung intrachromosomaler Wechselwirkungen in der Populationsdynamik von Drosophila. Verh. Dtsch. Zool. Ges. Heidelberg, Zool. Anzeiger, 31, Suppl.-Bd., 454-461.

Sperlich D., I967b. Populationsgenetik (Teil I, Drosophila). In: Fort-schr. Zool., 18, $223-278$.

SPERLICH D., FEUERBACH H., I969. Austausch-Ungleichgewicht zwischen unabhängigen Inversionen in natïrlichen Populationen von Drosophila subobscura. Theor. Applied Genetics, 39, ro4-II2.

Spertich D., KARLIK A., I968. Das Schicksal von markierten Letalchromosomen in mono- und polychromosomalen Experimentalpopulationen vòn Drosophila melanogaster. Verh. Dtsch. Zool. Ges. Innsbruck Zool. Anzeiger, 32, Suppl.-Bd., I95-205.

SPERLICH D., KARLIX A., I970. The genetic conditions in heterozygous and homozygous populations of Drosophila. I. The Fate of alien Chromosomes. Genetica, 41, 265-304.

SpIEss E.B., I968. Experimental Population Genetics. In: Annual Review of Genet., Annual Rev. Inc., Palo Alto.

TSChETWERIKOFF S.S. On certain features of evolutionary process from the view point of modern genetics. J. Exp. Biol. (Russian).

WALLACE B., I958. The average effect of radiation-induced mutations on viability in Drosophila melanogaster. Evolution, 12, 532-552.

WALlACE B., 1963. Further data on the overdominance of induced mutations. Genetics, 48, 633-65I.

Wallace B., 1968. Topics in Population Genetics. Norton and Co., New York.

WAllace B., DoBzhansky Th., 1962. Experimental proof of balanced genetic loads in Drosophila. Genetics, 47, 1027-1042.

WRIGHT S., DoBzHANSKy Th., 1946. Genetics of natural populations, XII. Experimental reproduction of some of the changes caused by natural selection in certain populations of Drosophila pseudoobscura. Genetics, 31, I25-I 56 . 